\title{
Análisis de la influencia de la altitud en los eventos de máxima precipitación en una cuenca del Pacífico: tendencias y variabilidad
}

\author{
Carlos Matovelle ${ }^{1,2}$, Bartolomé Andreo ${ }^{2}$ y Matías Mudarra² \\ (1) Grupo de Investigación en Geociencias, Ambiente y Recursos Naturales, Universidad Católica de Cuenca, Ecuador. \\ (correo-e: cmmatovelleb@ucacue.edu.ec) \\ (2) Departamento de Geología y Centro de Hidrogeología, Universidad de Málaga, España. (correo-e: andreo@uma.es; \\ mmudarra@uma.es).
}

Recibido Feb. 25, 2021; Aceptado Abr. 22, 2021; Versión final Jun. 20, 2021, Publicado Dic. 2021

\begin{abstract}
Resumen
El presente estudio busca determinar la influencia de la altura en los eventos de precipitación máxima diaria en la cuenca del Pacífico del río Jubones (Ecuador), que tiene una variación altitudinal muy marcada. Los eventos de precipitación son analizados basados en los percentiles 95 y 99 de la serie de datos pluviométricos, clasificándolos a su vez como eventos lluviosos y eventos extremadamente lluviosos. Los resultados muestran que la altura no influye en los eventos de precipitación diaria, incluyendo eventos lluviosos o muy lluviosos, cuando son evaluados como una serie total a lo largo del año. Sin embargo, si se utilizan indicadores robustos, se encuentra una relación entre altura y precipitación media. Esta relación está influenciada por la clasificación entre estación húmeda y estación seca. En conclusión, al analizar eventos muy lluviosos y extremadamente lluviosos como series diarias mensuales interanuales existe un alto coeficiente de correlación entre estos valores y los valores de altura.
\end{abstract}

Palabras clave: precipitación; eventos extremos; altitud; cuenca; Pacífico

\section{Analysis of altitude influence on maximum precipitation events in a Pacific basin: trends and variability}

\begin{abstract}
The present study seeks to examine the influence of altitude on maximum daily precipitation events in the Pacific basin of the Jubones River (Ecuador), which is known for having a marked difference in altitudinal variation. Precipitation events are assessed based on the 95th and 99th percentiles of a pluviometric data series, classifying them as rainy events and stormy events. The results show that altitude does not influence daily precipitation events, including rainy or very rainy, when events are taken as a total series throughout the year. However, if robust indicators are used for analysis, the results show a relationship between altitude and average rainfall. This relationship is affected by how the study area is classified between wet and dry seasons. In conclusion, examining very rainy and extremely rainy events as an inter-annual monthly daily series shows a high correlation coefficient between these values and altitude values.
\end{abstract}

Keywords: precipitation; extreme events; altitude; Pacific basin 


\section{INTRODUCCIÓN}

En términos generales la precipitación es un fenómeno natural que presenta una alta variabilidad espacio temporal en función de las características de cada área, consecuentemente esta variabilidad tiene impactos directos y significados en la hidrología de las cuencas (Chen et al., 2015). Se debe analizar esta variabilidad en la precipitación para conocer la disponibilidad del agua en una cuenca abastecedora de diversos usos de agua. Se sabe que, en determinadas zonas de estudio, existe una relación estadística entre los totales de lluvia integrados o promediados durante diferentes períodos de tiempo, con la elevación a la que se encuentre, esto hace posible aplicar métodos estadísticos de correlación para estimar las relaciones que existe entre las variables climáticas y geográficas (Leal et al., 2010). Adicional a esto, las herramientas tecnológicas actuales incorporan principios matemáticos para realizar análisis que brindan resultados con mayor exactitud y en menor tiempo, es por este motivo que en periodos recientes se hace posible realizar análisis que antes eran virtualmente imposible, debido al gran requerimiento de tiempo y procesamiento computacional, un ejemplo claro de este tipo de análisis son los Sistemas de Información Geográficos que emplean métodos como el de regresión ponderada geográficamente (Geographically Weighted Regression) GWR por sus siglas en inglés, que permite identificar, estimar y mapear las variaciones de la precipitación con respecto a la altura (Brunsdon et al., 2001).

En las cuencas hidrográficas que se encuentran en los Andes, la variabilidad de la topografía combinados con una notoria falta de datos dificulta los procesos de análisis de precipitaciones (Celleri et al., 2007), por lo tanto es fundamental buscar relaciones espacio-temporales que permitan conocer la variabilidad en estas cuencas. Para conocer la variabilidad suele utilizarse análisis estadísticos de las series de datos y realizarse ajustes mediante funciones adecuadas (Ávila-Parra y Martín-Vide, 2013); pero la variabilidad propia de cada región, condicionada por factores externos como corrientes de aire, vientos convectivos y factores morfológicos indican que cada vez es necesario buscar relaciones particulares para cada zona.

Tradicionalmente el coeficiente de correlación de Pearson es el más utilizado para estimar las relaciones existentes entre variables climáticas y variables geográficas (Kadiolu, 2000), sin embargo, la presente investigación aplica el coeficiente de correlación de Spearman, que describe la intensidad de la asociación monotónica entre los pronósticos y las observaciones (Fallas y Alfaro, 2012). Se resalta el hecho de que las diferencias entre los dos coeficientes son muy pequeñas, diferenciándose únicamente, en la mayoría de los casos, en los decimales del valor estimado por los coeficientes; pero el coeficiente de Spearman es una técnica no paramétrica con una distribución probabilística libre, esto permite que los supuestos sean menos estrictos pero robusto a la presencia de outliers (Bartholomew, 1995; Restrepo y González, 2007).

El objetivo principal de este estudio es determinar si existe relación entre la altura y los eventos de precipitación en la cuenca del río Jubones, partiendo de la relación establecida entre la temperatura y la altura conocida generalmente como gradiente térmico (Fritzsons et al., 2015). Para validar esta relación se han aplicado criterios estadísticos que determinen la variabilidad climática y la relación que existe entre las variables de altura y eventos de precipitación. Es así que, partiendo de la necesidad de verificar o estimar la relación entre los eventos de precipitación presentados a escala diaria, los eventos determinados como muy lluviosos y los eventos determinados como extremadamente lluviosos, respecto a la altura.

La cuenca del río Jubones está compuesta por varios pisos climáticos y ecológicos que comprenden desde el manglar hasta el páramo (Quintana et al., 2017), esta variación climática se da por ser una cuenca de la vertiente del Pacífico en Ecuador en donde es posible que el territorio de la cuenca nazca desde el Páramo y termine drenando sus aguas en el océano. Se tiene una variación climática, diferentes tipos de usos de suelo, varios aprovechamientos de agua y actividades económicas de gran interés de análisis. El comportamiento de la precipitación en la cuenca es heterogéneo y marcado tanto en intensidad como en comportamiento entre la planicie costera y la zona andina (Luna-Romero et al., 2018; Luna-Romero y Gaspari, 2018), este comportamiento no se ve reflejado en su totalidad en el comportamiento fluvial de los ríos ya que existen varias extracciones de agua para diversas actividades productivas en las diferentes zonas de la cuenca.

\section{METODOLOGÍA}

Este apartado está divido en la explicación del área de estudio con los respectivos datos utilizados para el análisis y posteriormente las condicionantes estadísticas que se han estudiado con el objeto de encontrar relaciones entre precipitaciones y altitud. En un primer análisis estadístico se busca correlaciones directas, luego se aplica estadísticos robustos y posteriormente se analiza los eventos máximos de precipitaciones clasificados de acuerdo a los cuartiles 95 y 99 como eventos lluviosos y extremadamente lluviosos respectivamente. 


\section{Área de estudio y data}

Se ha estudiado la cuenca del río Jubones por sus características particulares, como ser una cuenca de vertiente del Pacífico que juega un gran papel en la complementariedad hidrológica del Ecuador, sus características morfológicas particulares, los usos y presiones existentes sobre el agua. Las variaciones de clima de la cuenca cambian entre húmedo y árido, la precipitación puede variar en el año desde los $925 \mathrm{~mm}$ hasta los $290 \mathrm{~mm}$, pero existe una marcada disminución de la precipitación durante los meses de julio a diciembre, resultando un clima prácticamente desértico (Hasan y Wyseure, 2018).

La gestión del agua en el Ecuador y en la cuenca del Jubones históricamente ha cambiado desde ser una gestión centralizada a ir entregando las competencias a organizaciones de usuarios locales (Mills-Novoa et al., 2020), por este motivo toda investigación enfocada en conocer la variabilidad del recurso hídrico es gran importancia. En la Fig.1 se observa el área de la cuenca y las estaciones meteorológicas escogidas para el análisis propuesto:

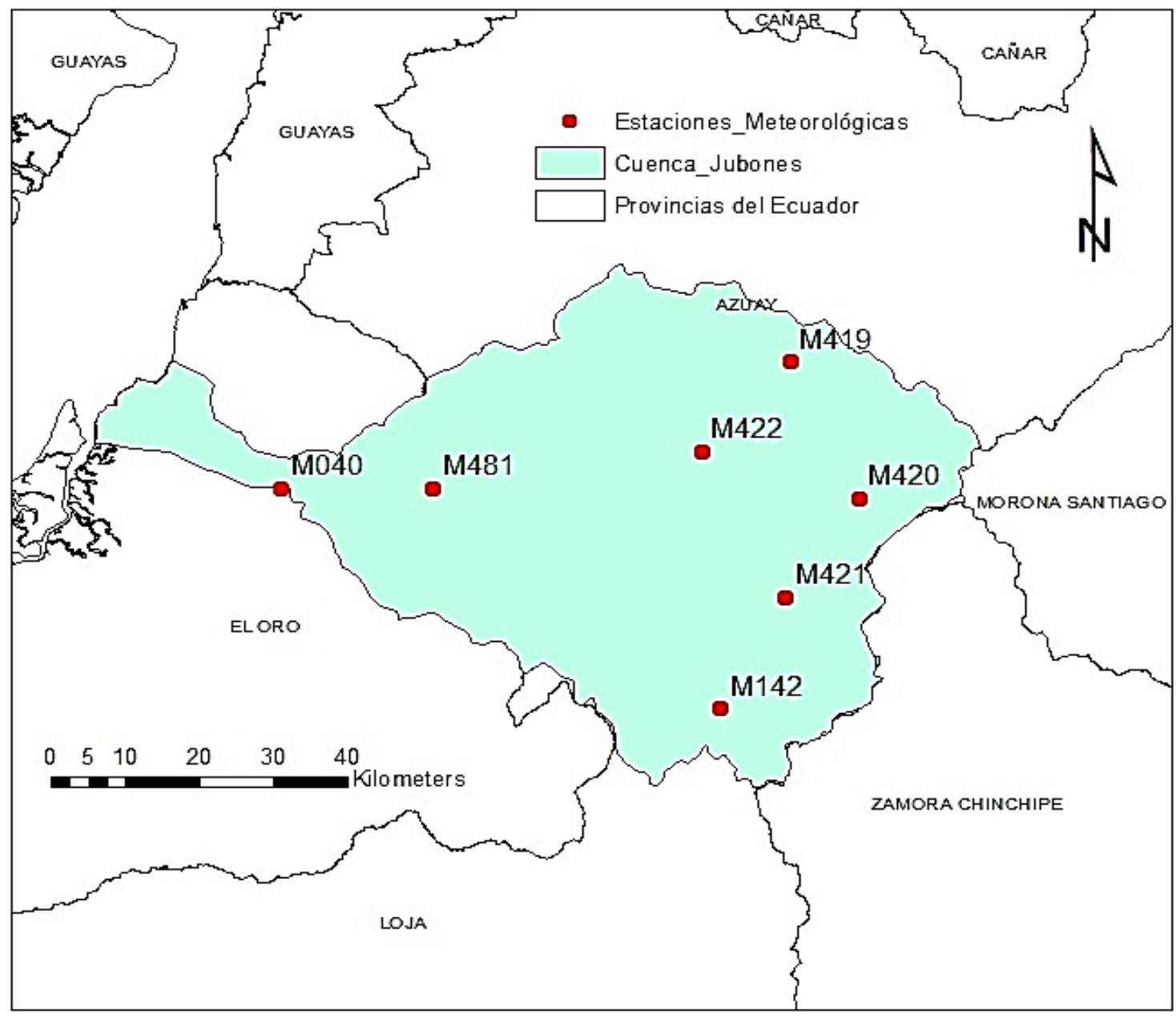

Fig. 1: Ubicación geográfica de las estaciones meteorológicas dentro de la cuenca de Jubones.

Las estaciones seleccionadas que se presentan en la figura anterior han sido el resultado de un análisis previo de validación de datos, quedando como resultados las que se encuentran en el mapa. Se puede observar que existe una distribución espacial adecuada que permite lograr resultados confiables de los cálculos realizados.

\section{Análisis estadístico}

El análisis estadístico se centra en los datos de precipitación obtenidos diariamente por las estaciones meteorológicas pertenecientes al INAMHI (Instituto Nacional de Meteorología e hidrología) de Ecuador, estaciones ubicadas dentro de la cuenca del Río Jubones, misma que comprende a las provincias de Azuay, Loja y El Oro, tal como se muestra en la Fig. 2. Esta cuenca se puede clasificar como altoandina debido a su limitación geográfica, parte desde las cercanías de la Cordillera andina hasta desembocar en la costa ecuatoriana, es decir tiene una variación altitudinal bastante marcada lo cual es favorable para este estudio. 
Estas estaciones meteorológicas poseen una serie de datos escala diaria que inicia en el año 1965 y termina en el año 2015, con datos cercanos a 40 años de registros meteorológicos, por lo que se considera que es un registro lo suficientemente largo para reflejar el comportamiento meteorológico de la zona de estudio. Debido a que se realiza un análisis de correlación entre altura y precipitación, es necesario que estas estaciones se encuentren en las partes alta, media y baja de la cuenca, por este motivo es necesario establecer estas zonas dentro de la cuenca. Para esto se emplea el Digital Elevation Model DEM recortado al perímetro de la cuenca para luego realizar tres quiebres en los datos del DEM, estos quiebres se determinaron mediante el método de pausas naturales o Jenks, ya que gracias a esta metodología las clases se basan en agrupaciones naturales inherentes a los datos, identificando los puntos de ruptura y seleccionando los saltos de clase que mejor agrupan valores similares y maximizan las diferencias entre clases, en este caso esta metodología permite delimitar de manera precisa tres zonas específicas de acuerdo a la altura.

La delimitación de las zonas alta-media-baja de la cuenca del Jubones, así como la distribución espacial de las estaciones meteorológicas de precipitación se muestran en la Fig.2. En esta misma gráfica se puede determinar que en la parte baja de la subcuenca se ubican dos estaciones meteorológicas, en la parte media se ubican tres estaciones meteorológicas y en la parte superior se encuentran dos estaciones meteorológicas, por lo que se dispone de una adecuada distribución de estaciones en el aspecto altitudinal de la cuenca, esto se detalla en la Tabla 1, en donde también se indica las alturas a las que se encuentran.

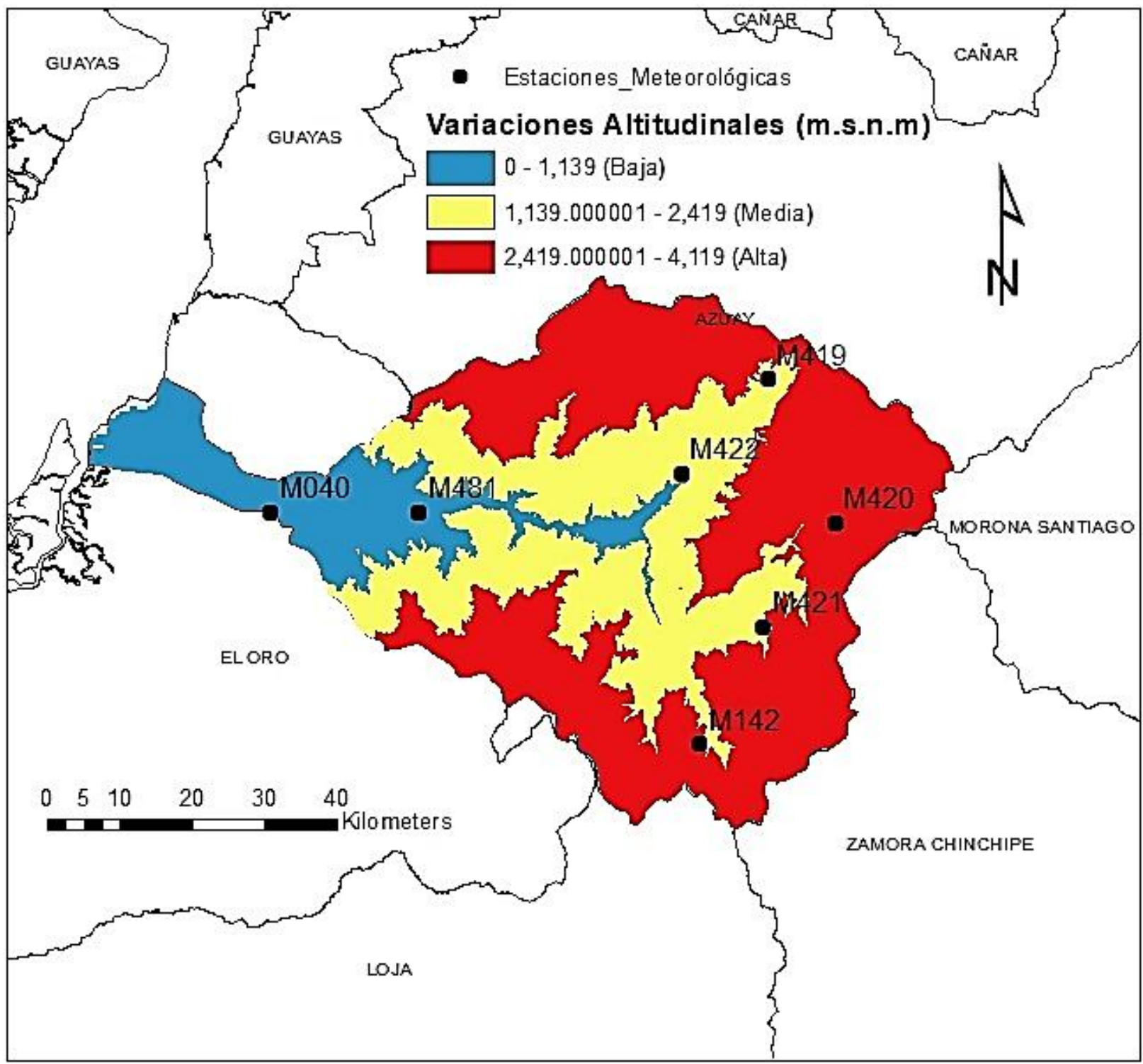

Fig. 2: Ubicación de las estaciones en las zonas alta media y baja de la cuenca del Jubones, de acuerdo a la clasificación altitudinal. 
Tabla 1. Ubicación de las estaciones meteorológicas de en las zonas alta-media-baja de la cuenca del Jubones

\begin{tabular}{|c|c|l|c|c|}
\hline Código & ID & Nombre & m.s.n.m & Zona \\
\hline M0040 & 1 & Pasaje & 40 & Baja \\
\hline M0481 & 2 & Ushcurrumi & 290 & Baja \\
\hline M0422 & 3 & $\begin{array}{l}\text { Hda. Santa Lucía - } \\
\text { Camino a Rircay }\end{array}$ & 1310 & Media \\
\hline M0419 & 4 & Girón & 2130 & Media \\
\hline M0421 & 5 & Oña & 2320 & Media \\
\hline M0142 & 6 & Saraguro & 2525 & Alta \\
\hline M0420 & 7 & Nabón INAMHI & 2750 & Alta \\
\hline
\end{tabular}

Una vez determinado que las estaciones meteorológicas poseen una distribución altitudinal y espacial que satisfacen los criterios para realizar la presente investigación se procede a realizar el análisis de dos criterios de interés establecidos: influencia de la altura sobre los eventos diarios de precipitación e influencia de la altura sobre la variabilidad climática determinada por eventos extremos los cuales serán determinados por el percentil 95 y 99 . El primer criterio nace de la curiosidad de establecer si existe una relación entre altura y precipitación, algo similar a la relación entre altura y temperatura, ya sea de forma directa o inversa. Similar al primer criterio de interés, el segundo criterio pretende analizar que, de no existir una relación en evento diario de precipitación respecto a la altura, se pueda encontrar una relación con los eventos muy lluviosos o extremadamente lluviosos respecto a la altura.

Para esto se propone la siguiente metodología, en un primer paso se obtiene el coeficiente de correlación de Spearman al establecer el valor de precipitación como variable dependiente y el valor de altura a la que se encuentra la estación como variable independiente, esto para cada uno de los días en el registro histórico de datos. Cabe resaltar que el uso del coeficiente de correlación de Spearman (r.s), a lo largo del presente análisis es debido a que se trabaja con datos de precipitación, en donde es recomendado el uso de este coeficiente (Habib et al., 2002).

Para datos que tengan distribuciones normales es común utilizar regresiones lineales y sus coeficientes pero es muy sensible a la presencia de atípicos, ahí es donde los métodos robustos se ven poco afectados por la presencia de estos outliers, entre los estimadores de regresión robustos se encuentran los LTS, LMS y los S estimadores (Cafaro et al., 2021), con este primer paso se logra determinar si existe una correlación de eventos diarios de precipitación respecto a la altura. Al ser una cuenca tan variable se propone el uso de la estadística robusta que es una aproximación alternativa a los métodos estadísticos clásicos. Estas técnicas se emplean con objeto de producir estimadores que no sean afectados por variaciones pequeñas respecto a las hipótesis de los modelos, esto podrá encontrar relaciones que no se vean tan claras con los análisis convencionales.

En un segundo paso se propone determinar los eventos superiores al percentil 95 y el percentil 99 , los cuales se clasifican como eventos o días lluviosos y extremadamente lluviosos respectivamente (Serrano Vincenti et al., 2012), una vez obtenidos estos resultados de eventos lluviosos y extremadamente lluviosos, los comparamos respecto a la altura de la estación a la que pertenece dicho registro, al igual que en el primer paso se obtendrán coeficiente de correlación de Spearman determinando si existe alguna relación entre estos eventos muy lluviosos y extremadamente lluviosos respecto a la altura.

\section{RESULTADOS Y DISCUSIÓN}

Conocer las precipitaciones extremas y su comportamiento resulta de gran interés científico y social, ya que permite gestionar los recursos hidrometeorológicos (Pineda y Willems, 2018). Para un mejor entendimiento y análisis de los resultados obtenidos por la metodología anteriormente descrita, los resultados se desglosarán mediante los criterios de interés.

\section{Influencia de la altura sobre los eventos de precipitación.}

Como se ejemplifica en la Figura 3, se toma a la altura como la variable independiente y al valor de la precipitación en cada uno de los días de la serie temporal como variable dependiente, de esta manera se obtiene el valor del coeficiente de correlación entre precipitación y altura para este día, luego, promediando los valores de coeficientes de correlación de todos los eventos, se obtuvo el valor de r.s= -0.0866 . 


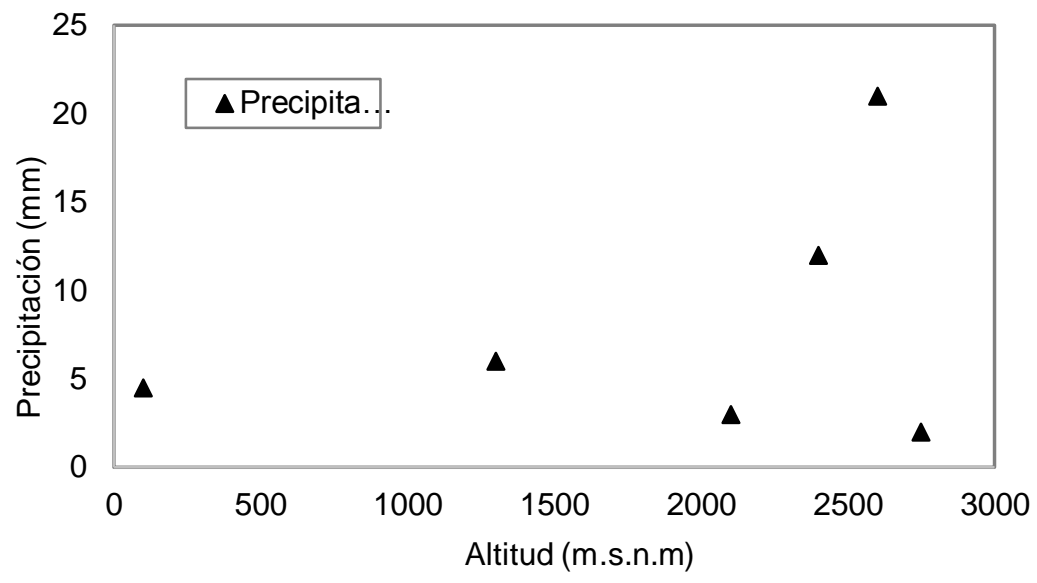

Fig. 3: Ejemplificación de comparación de evento con respecto a la altura.

Sin embargo, es necesario establecer una tabla clasificatoria o una tabla de interpretación de la fuerza de correlación mediante el coeficiente de correlación de Spearman, esto quiere decir que a un valor positivo o negativo cercano a 1, tendrían una muy fuerte correlación positiva o una muy fuerte correlación negativa respectivamente, tal como se muestra en la Tabla 2. Tomando esto en consideración y comparando la tabla clasificatoria respecto del valor obtenido, se puede concluir que existe una muy débil correlación negativa entre todos los eventos diarios de precipitaciones respecto a la altura.

Tabla 2. Interpretación de fuerza de correlación mediante coeficiente de correlación de Spearman. Adaptado de Martínez-Ortega et al., (2009).

\begin{tabular}{|l|l|}
\hline Valor del coeficiente r.s (positivo o negativo) & Significado \\
\hline 0.00 a 0.19 & Una muy débil correlación. \\
\hline 0.20 a 0.39 & Una débil correlación. \\
\hline 0.40 a 0.69 & Una moderada correlación. \\
\hline 0.70 a 0.89 & Una fuerte correlación. \\
\hline 0.90 a 1 & Una muy fuerte correlación. \\
\hline
\end{tabular}

En la Figura 4 se observa el ajuste más preciso logrado de los datos con una correlación de Spearman r.s de 0.25 , para el modelo LAD, lo cual indica una correlación débil. Con estas relaciones no existe manera de mejorar el ajuste para tener un indicador estadístico válido que indique una fuerte correlación de las variables. En la prueba LM se nota que existe un ligero incremento de la precipitación con la altura, pero el ajuste es muy pobre. Para mejora el modelo se trabaja con pruebas estadísticas robustas, obtenido un mejor modelo para los indicadores LTS, LMS y S. Todos estos indican relaciones negativas entre la precipitación y la altura, existiendo ajustes satisfactorios.

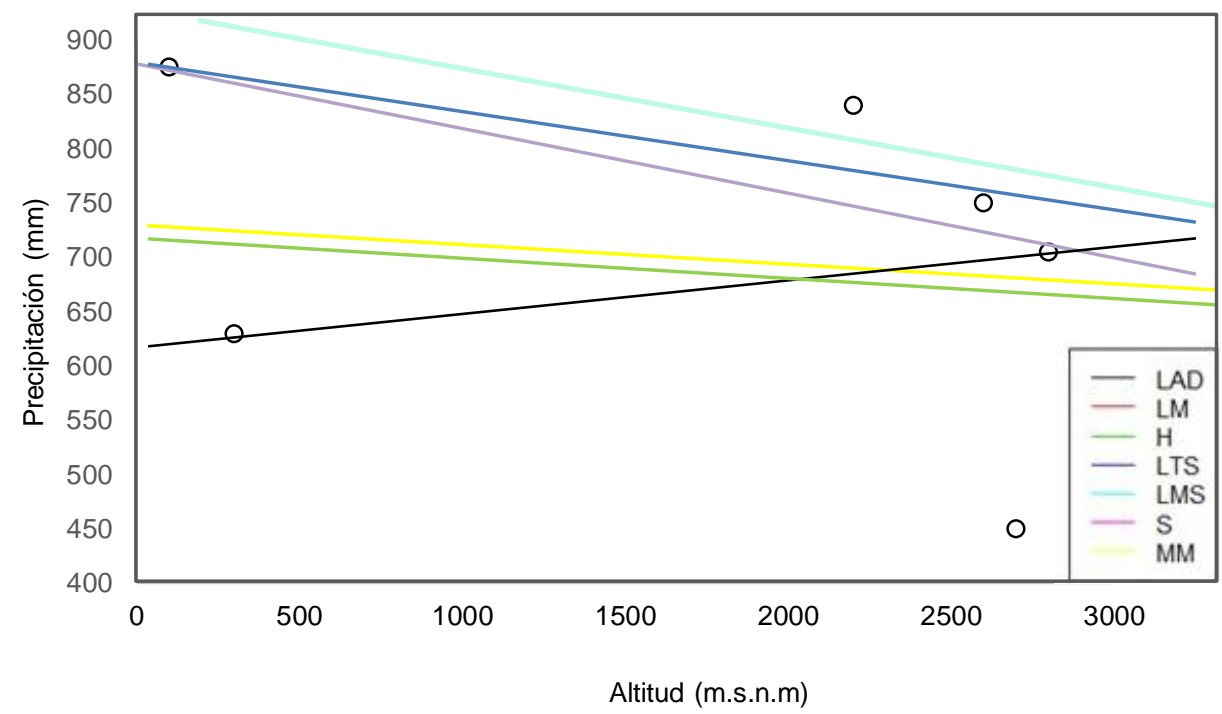

Fig. 4: Modelos robustos para la relación de precipitación media con la altura. 
Los modelos que guardan mayor similitud entre ellos son el LTS y el LMS, los cuales indican que la precipitación disminuye $4,8 \mathrm{~mm} / 100 \mathrm{~m}$ y $5,3 \mathrm{~mm} / 100 \mathrm{~m}$ respectivamente. Los análisis de la relación entre la altitud y la precipitación juegan un papel fundamental en los análisis hidrológicos y dentro de esto analizar los ciclos estacionales permitiría evaluar la verdadera relación que existe entre estas dos variables (Espinoza et al., 2010), de ahí la necesidad de realizar el siguiente análisis entre la altura relacionada con la variabilidad climática.

\section{Influencia de la altura sobre la variabilidad climática de precipitación.}

Al comparar los valores de los percentiles 95 y 99 de cada estación y al compararlas contra los valores de altura de las estaciones, se obtiene que el valor de correlación del percentil 99 frente a la altura es de r.s = 0.04 , lo cual significa que existe una muy débil correlación positiva entre la altura y el valor del percentil 99, ahora al obtener el valor de correlación del percentil 95 frente a la altura, se obtiene el valor de r.s $=-0.34$, lo cual significa que existe una débil correlación negativa entre altura y el valor del percentil 95 . Sin embargo al realizar una exploración más detallada de los datos, es decir clasificándolos y extrayendo estos percentiles 99 y 95 de cada una de las series diarias mensuales interanuales y al comparar estos datos de cada estación con la altura a la que se ubica la misma obtenemos los resultados mostrados en la Tabla 3 , y como se puede observar en la misma tabla existen valores de coeficiente de correlación muchos más altos de los que se tienen al comparar la serie diaria multianual de forma completa, ahora es interesante el cómo estos valores varían de valores positivos a valores negativos, por lo que al realizar un análisis clasificatorios de meses húmedos y meses secos en cada una de las estaciones en cada uno de los meses a lo largo del año determinados por su media total, obtenemos una curiosa relación en la que, dependiendo si se considera un mes húmedo o un mes seco los coeficientes de correlación de estos eventos lluvioso o extremadamente lluviosos tienen un valor positivo o negativo los cuales se clasifican entre una correlación moderada y una muy fuerte correlación ya sea positiva o negativa. En la Tabla 4 se presenta la caracterización de mes húmedo o seco, esto permite conocer la relación que tendrá la tendencia (positiva o negativa) para cada mes analizado.

Tabla 3. Valor de correlación entre altura y valor del percentil 95 y 99 para cada uno de las series diarias mensuales interanuales.

\begin{tabular}{|c|c|c|c|c|c|c|}
\hline Percentil & Enero & Febrero & Marzo & Abril & Mayo & Junio \\
\hline 95 & -0.46429 & -0.53571 & -0.53571 & -0.39286 & 0.78571 & 0.75679 \\
\hline 99 & -0.75 & -0.52254 & -0.60714 & -0.82143 & -0.53571 & 0.46429 \\
\hline Percentil & Julio & Agosto & Septiembre & Octubre & Noviembre & Diciembre \\
\hline 95 & 0.57143 & 0.67857 & 0.89286 & 0.92857 & 0.92857 & 0.42857 \\
\hline 99 & 0.71429 & 0.60714 & 0.82143 & 0.89286 & 0.78571 & -0.53571 \\
\hline
\end{tabular}

Tabla 4. Caracterización para cada una de las estaciones.

\begin{tabular}{|c|c|c|c|}
\hline Mes & \multicolumn{3}{|c|}{ Tipo de mes de acuerdo a las estaciones analizadas } \\
\hline Enero & \multicolumn{3}{|c|}{ Mes húmedo } \\
\hline Febrero & \multicolumn{3}{|c|}{ Mes húmedo } \\
\hline Marzo & \multicolumn{3}{|c|}{ Mes húmedo } \\
\hline Abril & \multicolumn{3}{|c|}{ Mes húmedo } \\
\hline Mayo & Mes seco & \multicolumn{2}{|c|}{ Mes húmedo (De acuerdo a la estación M0422) } \\
\hline Junio & \multicolumn{3}{|c|}{ Mes seco } \\
\hline Julio & \multicolumn{3}{|c|}{ Mes seco } \\
\hline Agosto & \multicolumn{3}{|c|}{ Mes seco } \\
\hline Septiembre & \multicolumn{3}{|c|}{ Mes seco } \\
\hline Octubre & \multicolumn{3}{|c|}{ Mes seco } \\
\hline Noviembre & Mes seco & \multicolumn{2}{|c|}{ Mes Húmedo (De acuerdo a la estación M0420) } \\
\hline Diciembre & \multicolumn{2}{|c|}{ Mes seco (De acuerdo a las estaciones M0040, M0422) } & Mes húmedo \\
\hline
\end{tabular}


En la Figura 5 (A) se puede ver la tendencia para una estación en un mes seco y en (B) para un mes húmedo en donde se aprecia claramente la tendencia que existe con una correlación que se podría clasificar como fuerte para este caso específico. Existe estudios similares que indican tendencias de precipitación entre un rango de valores, como (Schatz Sá et al., 2017) que analiza una tendencia creciente entre eventos mayores a $50 \mathrm{~mm}$ y $100 \mathrm{~mm}$, sin embargo, existen otros como que no encuentran relaciones entre el análisis de pluviómetros de una zona particular (Silva, 2018), de ahí la importancia de este tipo de estudios a nivel regional para encontrar relaciones particulares en cada zona que permitan una gestión adecuada del recurso. La variabilidad climática de la precipitación generalmente tiene una tendencia en aumento (Revueltas et al., 2020) y en la investigación se puede analizar que los efectos de precipitaciones intensas tienen cumplen esta relación en los meses húmedos que se caracterizan por ser los meses donde se dan particularmente estas lluvias intensas.

En el estudio de Suárez-Aguilar et al., (2020) se aplican modelos matemáticos específicos para obtener el comportamiento de la precipitación debido a su alta variabilidad, razones por las cuales se evidencia que para cada zona puntual existen características particulares que deben ser abordas con todas las herramientas matemáticas y estadísticas disponibles para intentar que los resultados se asemejen a la realidad con un nivel de confiabilidad aceptable.

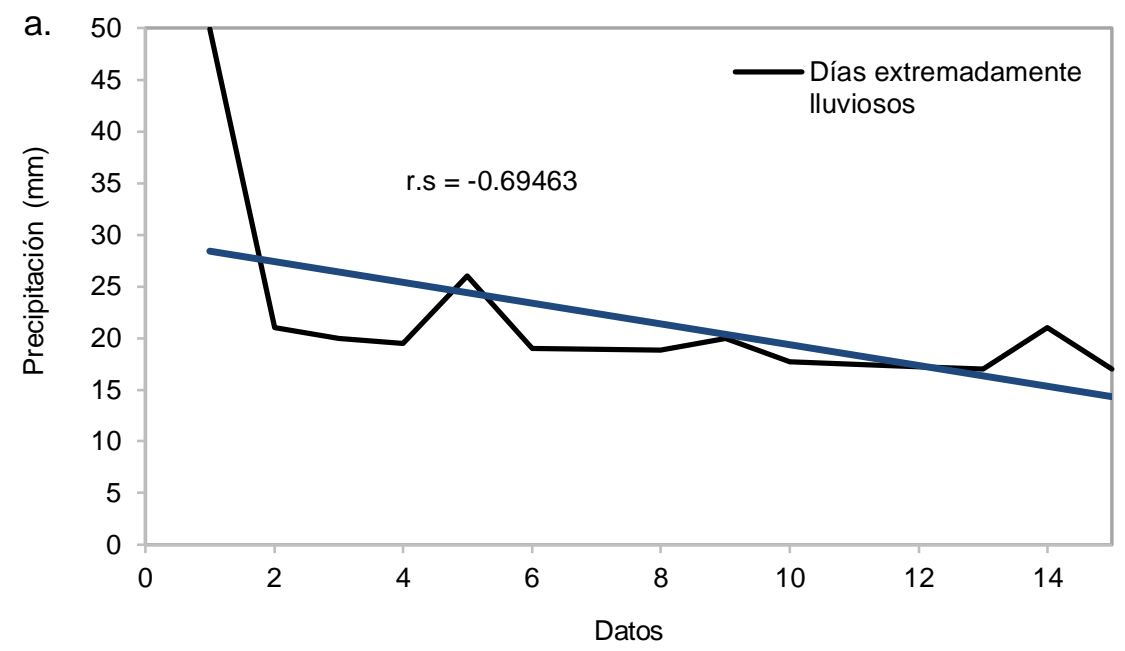

b.

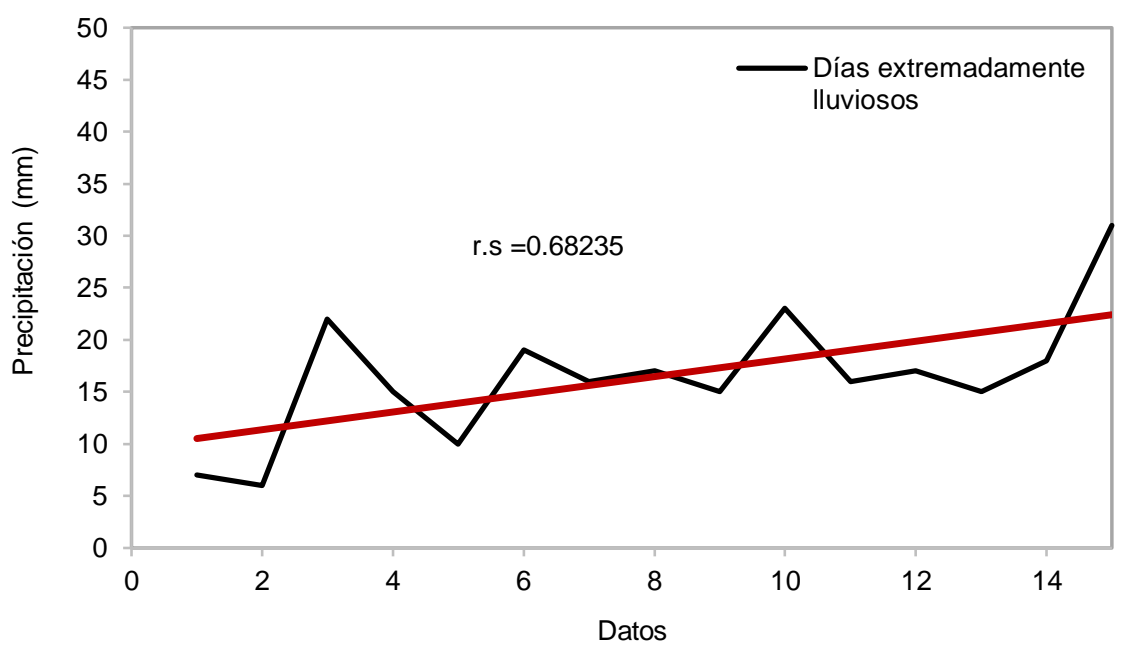

Fig. 5: Ejemplo de dos correlaciones fuertes para estaciones en diferentes meses típicos.

\section{DISCUSION FINAL}

En base a los resultados previos los eventos diarios de precipitación no se ven influenciados casi de manera significativa por la altura, esto quiere decir que como modelo predictor, como modelo de relleno de datos la altura no permite estimar los valores de precipitación en eventos diarios. Sin embargo, si se realiza un análisis diario mensual interanual para para obtener la relación entre la altura y los eventos extremos de precipitación catalogados como lluviosos y extremadamente lluviosos, podemos concluir que dependiendo en qué época del año ya sea húmeda o seca, es decir, la época húmeda que abarca desde diciembre - enero a los meses de abril 
- mayo presenta una correlación negativa que se va desde débil a muy fuerte al evaluar los eventos lluviosos y extremadamente lluviosos, la época seca que abarca desde mayo - junio a los meses de noviembre - diciembre presenta una correlación positiva que se va desde moderada a muy fuerte al evaluar los eventos lluviosos y extremadamente lluviosos, así podemos concluir además que, en los meses de transición entre época seca y época húmeda, es donde se presentan los valores más pequeños ya sean positivos o negativos de este coeficiente de correlación.

Es interesante el obtener esta correlación ya que nos permite estimar de cierta forma los eventos extremadamente lluviosos o muy lluviosos, los cuales pueden desencadenar impactos naturales como son las inundaciones, lo cual puede traer a su vez perdida de cultivos e infraestructura, y lograr extrapolar esta información a toda la cuenca tomando como referencia la altura a la que se encuentra la zona de interés o la zona a la que se quiere realizar este estudio. Al comparar los valores de los resultados finales podemos determinar que existe una relación negativa muy baja, por lo que se determina que la altura prácticamente no tiene ninguna influencia sobre los eventos de precipitación a escala diaria dentro de la cuenca del Jubones. Al ser la metodología de GWR, un modelo de regresión múltiple que permite evaluar la presencia de diferentes factores que relacionen diferentes puntos del espacio, se podría, en futuros análisis evidenciar la influencia de otros factores diferentes a la altura, los cuales condiciones a los eventos de precipitación, enfocándose a diferentes propósitos de análisis (Brunsdon et al., 2010).

\section{CONCLUSIONES}

De acuerdo al trabajo presentado y a los resultados obtenidos, se puede plantear que no existe una correlación directa indicada por el coeficiente de Spearman entre los datos de precipitaciones diarias y la altitud de la cuenca, los valores de r.s indican un ajuste pobre de la comparación. Cuando se emplean modelos de estadística robusta los indicadores mejoran su ajuste, obteniendo relaciones inversas entre la altitud y la precipitación mediante dos modelos específicos que son LTS y el LMS. Estos modelos indican que la precipitación disminuye a medida que aumenta la altitud, para la zona baja es en donde podemos encontrar valores más elevados de precipitaciones diarias.

Para los eventos extremos indicados con los percentiles 95 y 99 el análisis muestra correlaciones con un nivel de ajuste más alto, pero existen tendencias directamente proporcionales de los eventos máximos de precipitaciones de acuerdo a la caracterización de la estación. Cuando observamos épocas húmedas las relaciones son positivas, indicando que los eventos se incrementan con la altura y para las épocas marcadas por secas en las estaciones la correlación es inversamente proporcional, para este caso existen eventos más altos en la zona baja de la cuenca en la época seca. Estos resultados dan una idea más clara, dentro de la gran variabilidad de las precipitaciones, del comportamiento que puede tener los eventos de lluvia en cada época del año, entendiendo de mejor manera las ocurrencias que puede haber en el tiempo y en el espacio marcado por las series de las estaciones analizadas.

\section{REFERENCIAS}

Ávila-Parra, K., y Martín-Vide, J., Análisis estadístico de los eventos extremos de precipitación en la zona centro y sur de Chile continental, https://doi.org/10.30827/cuadgeo.v52i1.926, Cuadernos Geográficos, 52(1), 69 - 83 (2013).

Bartholomew, D. J., Spearman and the origin and development of factor analysis, https://doi.org/10.1111/j.20448317.1995.tb01060.x, British Journal of Mathematical and Statistical Psychology, 48(2), 211-220 (1995).

Brunsdon, C., Fotheringham, A. S., y Charlton, M. E., Geographically Weighted Regression: A Method for Exploring Spatial Nonstationarity, https://doi.org/10.1111/j.1538-4632.1996.tb00936.x, Geographical Analysis, 28(4), 281-298 (2010).

Brunsdon, C., Mcclatchey, J., y Unwinm D.J., Spatial variations in the average rainfall-altitude relationship in Great Britain: an approach using geographically weighted regression, https://doi.org/10.1002/joc.614, International Journal of Clumatology, 466, 455-466 (2001).

Cafaro, M., Melle, C., Pulimeno, M., Epicoco, I., Fast online computation of the Qn estimator with applications to the detection of outliers in data streams, https://doi.org/10.1016/j.eswa.2020.113831, Expert System Applied, 164 (2021).

Celleri, R., Willems, Pa., Buytaert, W., y Feyen, J., Space-time rainfall variability in the Paute Basin, Ecuadorian, https://doi.org/10.1002/hyp.6575 Space-time, Hydrological Processes, 2274, 2267-2274, (2007).

Chen, Y., Liu, H., An, J., Görsdorf, L., y Berger, F. H., A field experiment on the small-scale variability of rainfall based on a network of micro rain radars and rain gauges, https://doi.org/10.1175/JAMC-D-13-0210.1, Journal of Applied Meteorology and Climatology, 54(1), 243-255 (2015).

Espinoza, J. C., Ronchail, J., y otros seis autores, Variabilidad espacio-temporal de las lluvias en la cuenca amazónica y su relación con la variabilidad hidrológica regional. Un enfoque particular sobre la región andina, Revista Peruana GeoAtmosférica RPGA, 130(2), 99-130 (2010). 
Fallas, B., y Alfaro, E., Uso de herramientas estadísticas para la predicción estacional del campo de precipitación en América Central como apoyo a los Foros Climáticos Regionales: Análisis de Correlación Canónica, Revista de Climatología 12, 93-105 (2012).

Fritzsons, E., Wrege, M., y Mantovani, E., Altitude e temperatura: estudo do gradiente térmico no Rio Grande do Sul, https://doi.org/http://dx.doi.org/10.5380/abclima.v16i0.39665, Revista Brasileira de Climatologia, 16, 108-109 (2015).

Habib, E., Krajewski, W. F., y Ciach, G. J., Estimation of Rainfall Interstation Correlation, https://doi.org/10.1175/15257541(2001)002<0621:eoric>2.0.co;2, Journal of Hydrometeorology, 2(6), 621-629 (2002).

Hasan, M. M., y Wyseure, G., Impact of climate change on hydropower generation in Rio Jubones Basin, Ecuador, https://doi.org/10.1016/j.wse.2018.07.002, Water Science and Engineering, 11(2), 157-166 (2018).

Kadiolu, M., Regional variability of seasonal precipitation over Turkey, https://doi.org/10.1002/10970088(20001130)20:14<1743::AID-JOC584>3.0.CO;2-G, International Journal of Climatology, 20(14), 1743-1760 (2000).

Leal-Mendoza, M., y Gonzalez, E., Spatial analysis and modeling of climate variables in the Cuitzeo Basin, Mexico. Investigaciones Geograficas, 72(8701), 49-67(2010).

Luna-Romero, A., y Gaspari, F. J., Variabilidad de la precipitación en la cuenca del río Jubones, XXVIII Congreso Latinoamericano de Hidráulica, 45-47 (2018).

Luna-Romero, A., Ramírez, I., y otros cuatro autores, Spatio-temporal distribution of precipitation in the Jubones river basin, Ecuador, https://doi.org/10.17268/sci.agropecu.2018.01.07, Scientia Agropecuaria, 9(1), 63-70 (2018).

Martínez-Ortega, R.M., Tuya, L.C., y otros tres autores, El coeficiente de correlación de los rangos de Spearman caracterización, Revista Habanera de Ciencias Médicas, 8 (2), 1 -19 (2009).

Mills-Novoa, M., Boelens, R., Hoogesteger, J., y Vos, J., Governmentalities, hydrosocial territories \& recognition politics: The making of objects and subjects for climate change adaptation in Ecuador, https://doi.org/10.1016/j.geoforum.2020.06.024, Geoforum, 115(July), 90-101 (2020).

Pineda, L. E., y Willems, P., Rainfall extremes, weather and climate drivers in complex terrain: A data-driven approach based on signal enhancement methods and EV modeling, https://doi.org/10.1016/j.jhydrol.2018.05.062, Journal of Hydrology, 563, 283-302 (2018).

Quintana, C., Pennington, R. T., Ulloa, C. U., y Balslev, H., Biogeographic Barriers in the Andes: Is the AmotapeHuancabamba Zone a Dispersal Barrier for Dry Forest Plants?, https://doi.org/10.3417/d-17-00003a, Annals of the Missouri Botanical Garden, 102(3), 542-550 (2017).

Restrepo, B. L., y González, L. J., De Pearson a Spearman. Revista Colombiana de Ciencias Pecuarias, 20, 183-192. (2007).

Revueltas, J. E., Zabaleta, A., Mercado, T., y Aguirre, S., Cambios en el clima local y su efecto en la regulación hídrica en microcuencas del departamento del Magdalena, Norte de Colombia, https://doi.org/10.4067/s071807642020000600193, Información Tecnológica, 31(6), 193-206 (2020).

Schatz Sá, E. A., Natel de Moura, C., Padilha, V. L., y Camargo Campos, C. G., Trends in daily precipitation in highlands region of Santa Catarina, southern Brazil, https://doi.org/10.4136/1980-993X, Revista Ambiente e Agua, 13(31), 1-13 (2017).

Serrano, S., Zuleta, V., y otros cuatro autores, Análisis estadístico de datos meteorológicos mensuales y diarios para la determinación de variabilidad climática y cambio climático en el Distrito Metropolitano de Quito, https://doi.org/10.17163/lgr.n16.2012.03, La Granja, 16(2), 23-47 (2012).

Silva, K., Future irrigation water requirements in the ljuí River basin, RS, http://dx.doi.org/10.1590/18071929/agriambi.v22n1p57-62, Revista Brasileira de Engenharia Agrícola e Ambiental, 22(1), 57-62 (2018)

Suárez-Aguilar, Z., Sepúlveda-Delgado, O., Patarrayo-Meza, M., Canaria-Camargo, L., Modelo matemático para estimar curvas de intensidad, duración y frecuencia de lluvias extremas en Tunja, Colombia, Información Tecnológica, 31(1), 193-206 (2020). 